American Medical Journal 1 (1): 23-26, 2010

ISSN 1949-0070

(C) 2010 Science Publications

\title{
A Comparative Study between Norethisterone Progestogens and Dydrogesterone in the Treatment of Dysfunctional Uterine Bleeding
}

\author{
Nabila K. Yaaqoub \\ Department of Gynaecology and Obstetrics, Faculty of Medicine, \\ University of Tikrit, P.O. Box 42, Tikrit Salahuddin, Iraq
}

\begin{abstract}
Problem statement: To compare the effects of both Norethisterone Progestongens and Dydrogesterone in the treatment of Dysfunctional Uterine bleeding Design: A prospective study. Setting: Tikrit Teaching Hospital, Iraq. Approach: About 200 patients presented with abnormal uterine bleeding and diagnosed as dysfunctional uterine bleeding were included in this study. Group A (study group): Include 100 patients were treated with norethisterone progestogens. Group B (control group): Include 100 patients were treated with dydrogesterone the response of the patients were assessed by regularity of menstrual cycle. Results: About 75 patients had regular cycle while 25 patients still had irregular bleeding regarding first group. 46 patients had regular cycle while 25 patients still had irregular bleeding regarding second group, decrease in severity of bleeding and resumption of regular cycle were more apparent with Norethiserone drug. Conclusion/Recommendations: Both norethisterone progestogens and dydrogesterone can be used in the treatment of dysfunctional uterine bleeding. Norethisterone progertogens showed higher rates of regular resumption of menses than Dydrogesterone and I recommended to use Norethisertone in the treatment of other problems like regulate ovulation in infertile woman.
\end{abstract}

Key words: Norethisterone progestogens, dydogestrone dysfunction uterine bleeding, menorrhagia, anovulatory cycle, menstruation

\section{INTRODUCTION}

Dysfunctional Uterine Bleeding (DUB): Is best define as abnormal bleeding from the uterus in the absence of organic disease of the genital tract and applies to any abnormal uterine bleeding, including disturbances of the menstrual cycle, regular and irregular uterine bleeding and alterations in the amount or duration of menstrual loss, but most commonly implies excessive regular menstrual bleeding or essential menorrhagia (Van, 1995a).

DUB is a group of disorders characterized by dysfunction of uterus, ovary, pituitary, hypothalamus or other part of the reproductive system, which results in abnormal or excessive uterine bleeding and the diagnosis is usually made by exclusion of organic disease of the genital tract (Davey, 1995).

Abnormal uterine bleeding in general due to organic and non organic causes, the organic causes due to sub mucous fibroid, Adenomyosis, endometrial and end cervical polyp, pelvic inflammatory disease and malignanet disease (Stuartc, 2000).

Classification of DUB:

Primary: Due to primary dysfunction in the uterus, ovary, pituitary, hypothalamus or higher centers.
Secondary: To either:

- Intrauterine contraceptive device or administration of sex hormones for contraception or other purposes

- Organic disease outside the reproductive system (Jacobs, 1995)

Twelve percent of all gynecological problems are due to menorrhagia and the commonest cause for hysterectomy (Charles, 1999).

The duration of menstrual blood loss varies normally in different women from 2-7 days with a mean of 5 days, but any menstruation lasting 8 days or longer should be regarded as excessive (Van, 1995b).

The best measure of the amount of menstruation is the total Menstrual Blood Loss (MBL), which is estimated from the total hemoglobin extracted from all tampons, towels and other material used during menstruation and measured objectively by alkaline haematin or other standard method (Hallberg, 1995).

DUB can occur at any age; though it's an etiology and management vary greatly in different age groups so an understanding of the effect of age and parity on management and on the risk of missed uterine pathology is important (Van, 1995b). 
The years immediately following the menarche are characterized by irregular menstrual cycles and long cycles due to immaturity of the hypothalamus and pituitary and menstrual cycles may be a novulatory, the middle years of reproductive life in normal women are characterized by regular menstrual cycles and regular ovulation, in premenopausal years menstrual cycles often become irregular again due to the decreased number of ovarian follicles and their increased resistance to gonadotrophin stimulation, this results in a progressive increase in corpus lustrum insufficiency or anovulatory cycles and eventually in cessation of menstruation (Suther Land, 1995).

History and examination are important in the diagnosis of DUB, a full blood count is an essential investigation in a patient with abnormal bleeding, thyroid function tests to exclude thyroid diseases, midluteal progesterone level test is performed when patient has a regular cycle only, a level greater than $30 \mathrm{nmol} \mathrm{L}^{-1}$ is indicative of ovulation, prolactin level because increase level result in an ovulation that cause abnormal uterine bleeding, coagulation screen is important if a bleeding disorder is suspected, serum androgens may be elevated in patients with polycystic ovarian syndrome (Geeta, 2000).

Transvaginal ultrasound is an excellent tool for evaluating pelvic structures and pathology, new developments with Doppler ultrasound will provide information on pelvic vascularity while 3-D ultrasound will aid the diagnosis of congenital uterine abnormalities.

Hysteroscopy is the gold standard procedure as it provides visualization of entire uterine cavity, is ideally performed during the proliferative phase of the menstrual cycle when the endometrium is at its thinnest (Jane, 2000).

In acute situation the main priorities of treatment involve, correction of anemia and arresting ongoing bleeding.

Progestogens are mainly indicated in patients with anovulatory bleeding, to reverse the effects of estrogenmediated endometrial proliferation and induce endometrial maturation.

Others Drugs can be used in DUB like combined oral contraceptives, danzol, gonadotrophin releasing hormone analogues like buserelin and goserelin and levenorgestral-releasing intra-uterine contraceptive device (mirena).

Other agents like antifibrinolytics like tranexamic acid and non-steroidal anti-inflammation agents like mefenamic acid can also arrest bleeding (Jane, 2000).

Dilatation and curettage can reduce bleeding in 75$80 \%$ of cases in acute situation, endometrial resection and ablation by diathermy and laser can reduce bleeding in $40 \%$ and finally hysterectomy as option for patients who have not responded to medical therapy or to more conservative surgical options.

Both laparoscopic myolysis and embolization techniques have been reported to be successful modes of treatment (Farn, 2000).

Primolut $\mathrm{N}$ can be used in dysfunctional bleeding, primary and secondary amenorrhea, premenstrual syndrone, mastopathy and endometriosis.

In rare cases nausea may occur, it's contraindicated in pregnancy, sever disturbances of liver function, dubin-johuson syndrome, previous or existing liver tumors.

A history of jaundice or severe pruritus during pregnancy, a history of herpes of pregnancy and thrombo-embolic processes.

If migrainous headaches or unusual severe headaches, sudden disturbances of vision or hearing, unusual pains or swelling of the legs, stabbing pains on breathing, significant rise in blood pressure, onset of jaundice or itching or hepatitis there is should be discontinuation of the drug.

Recent investigations have confirmed that norethisterone is partly metabolized to ethinylestradiol it's c-19 derivative that cause more androgenic sideeffects such as acne and greasy skin than C21 derivatives (Aladin and Yousif, 1990).

Dydrogesterone (duphaston): Is C21 derivatives cause less androgenic side-effects but more psychological distress such as anxiety, appear to have less effect than C19 derivatives on lipoprotein (Whitehead, 1999).

\section{MATERIALS AND METHODS}

This study was conducted on women attending gynecological unit in Tikrit teaching hospital over a period of one year from October 2008 to October 2009, it is a prospective study.

The total number of women included in this study were 225,25 women withdrew from the study due to intolerance of the side effects of the drugs, 200 women were the total patients included in this study, 100 were controls and 100 were the study group.

None of those patients were treated with ant bleeding agents in the previous 3 weeks.

A full history was obtained from each woman and a full physical examination was performed, the patients were divided into 2 groups:

- Group A (Study group): Include 100 patients were treated with norethisterone progestogens 
- Group B (control group): Include 100 patients were treated with dydrogesterone

The patients in both groups were diagnosed as dysfunctional uterine bleeding after exclude the organic pathology by careful history taken from the patients about the attach of bleeding, severity, duration and associated symptoms, any associated gynecological problems including dysmenorrheal, infertility, menopausal symptoms, any symptoms suggestive of bleeding disorder or myxoedema.

History of administration of sex hormones, intrauterine contraceptive device, history of heparin or wayfaring using, pelvic inflammatory disease and organic pathology outside the reproductive system like hemorrhoid, anal fissure that causing bleeding per rectum or haematuria due to renal stone for example and miss diagnosed as vaginal bleeding.

General, pelvic and abdominal examination are essential, general examination for stigma of systemic diseases like hirsutisum, strait, thyroid enlargement,skin pigment changes for echymosis, petechi, examination of lymph nodes, liver and spleen.

Inspection of vulva for bleeding, infection and speculum examination of vagina and cervix were essential for exclude any pathology.

Ultrasound examination was performed for both groups to exclude pelvic mass or possible pregnancy complications, fibroids, ovarian cyst, endometrial polyp and measurement of endometrial thickness.

Also Baseline Hemoglobin ( $\mathrm{Hb}$ ) was done for both groups as a part of assessment of the severity of the bleeding.

All patients were in reproductive age group between 20-45 years old and all were married, from the ethical point of view all patients were asked if they would like to participate in the study and a full explanation about the nature and side effects of the drugs, if the patient presented with sever uterine bleeding can be arrested by large doses of progestogens, norethisterone acetate (primolut N) 20-30 mg daily is given until bleeding stops (within 24-48 h) and for not more than 3 days, then the progestogens may continued at lower dose for 21 days, if not sever bleeding, norethisterone acetate $5 \mathrm{mg}$ daily from the 5th-25th day of cycle and continued for a minimum of 3 cycles, this protocol for the first group (Group A).

The protocol for the second group (Group B),the bleeding can be arrested by given $10 \mathrm{mg}$ dydrogesterone (duphaston) one Tablet daily 3 times over 10 days, then continued at lower dose for 21 days, if not sever bleeding dydrogesterone $10 \mathrm{mg}$ daily from 5th-25th day of cycle and continued for a minimum of 3 cycles.
Table 1: Character of patient

\begin{tabular}{lcl}
\hline Groups & Study group & Control group \\
\hline Number of patients & 100 & 100 \\
Age $($ mean \pm SD) & $28 \pm 3.5$ & $27.5 \pm 3.8$ \\
\hline
\end{tabular}

Table 2: Response of patient according to type of drugs

\begin{tabular}{lccc}
\hline Effects & $\mathrm{R} /($ primolut $\mathrm{N})$ & $\mathrm{R} /($ duphaston $)$ & Total \\
\hline $\begin{array}{l}\text { Stop bleeding with } \\
\text { regular cycle }\end{array}$ & 75 & 46 & 121 \\
$\begin{array}{l}\text { Not stop bleeding } \\
\text { Total }\end{array}$ & 25 & 54 & 79 \\
\hline
\end{tabular}

For both groups we gave one Tablet primolut $\mathrm{N}$ or dydrogesterone 2 times daily from the 19th-26th day of cycle as prophylactic dose after we control the bleeding to prevent recurrence of dysfunctional bleeding for at least 3 cycles.

\section{RESULTS}

The all patients involve in this study were married and in reproductive age group (20-45), so both groups were matched for the age and it was founded that there is no significant difference between mean age of both group.

Group A (study group): Mean age $\pm \mathrm{SD}=28 \pm 3.5$ (SD (Standard deviation)

Group B (control group): $27.5 \pm 3.8$ when applying ttest:

$$
\mathrm{T}=1.86, \text { so } \mathrm{p}<0.05
$$

No significant difference between both groups regarding the age as in Table 1.

In the study group which is on primolut $\mathrm{N}$ there is 75 patients had regular cycle with arrest bleeding within a period of treatment, while 25 patients still had irregular cycle with no control bleeding.

In the control group which is on duphaston there is 46 patients had regular cycle with arrest bleeding, while 54 patients still had irregular cycle with no control bleeding as in Table 2:

$$
\begin{gathered}
\text { Chi-Square }\left(\mathrm{X}^{2}\right)=18.4 \\
\mathrm{df}=\text { Degree of freedom } \\
\mathrm{p}>0.05 \text { (probability) }=\text { Level of significant }
\end{gathered}
$$

There is a very significant difference between both groups according to the type of treatment.

\section{DISCUSSION}

The most common cause of abnormal uterine bleeding in premenopausal woman is estrogenized an ovulation (Steve, 2003).

Menorrhagia affects approximately $15-20 \%$ of women presenting with abnormal uterine bleeding and 
cause significant social inconvenience as well as the potential for significant anemia (James, 2003).

Unfortunately, there is no simple clinical way to quantities the amount of blood lost during a menstrual period, counting the number of sanitary protective's used in a menstrual cycle has proven to be inaccurate based on differences in personal hygiene and cultural back grounds, when accurately quantities, blood loose during an entire normal adulatory menstrual cycle is approximately $60-80 \mathrm{~mL}^{-1}$, this amount of blood loose will not cause a decrease in hemoglobin in women consuming normal balanced diet (Beth, 2003).

As described by Arthur (2003), dysfunctional uterine bleeding unrelated to mechanical factors, iatrogenic causes, infectious agents, cancer or pregnancy.

They're no one particular pattern of bleeding that unequivocally defines dysfunctional uterine bleeding (Arthur, 2003).

All women in our study were in the reproductive age group and there is no significant difference between study and control groups regarding age as in Table 1.

About 75 patients were putted on primolut $\mathrm{N}$ Tablet had regular cycle and stop their bleeding while 25 patients still had irregular bleeding, which is consistent with the findings of Davey (1995).

About 46 patients were putted on duphastone Tablet had regular cycle and stop their bleeding while 54 patients still had irregular bleeding, which is consistent with findings of Charles (1999).

So there is a significant difference between both groups according to the type of treatment which is consistent with findings of Matthew (2003).

\section{CONCLUSION}

- Both norethisterone progestogens and dydrogesterone can be used in the treatment of dysfunctional uterine bleeding

- Norethisterone progestogens were more effective in the treatment of dysfunctional uterine bleeding than dydrogesterone

\section{REFERENCES}

Aladin, A.S. and Z. Yousif, 1990. Iraqi Drug Guide. 1st Edn., John Wiley and Sons, pp: 168-170.

Arthur, F., 2003. Hormones and Abnormal Uterine Bleeding. Danforth's Obstetrics and Gynecology. 9th Edn., Lippincott Williams and Wilkins, ISBN: 0-3452-5430-1, pp: 645-646.

Beth, Y., 2003. Abnormal Uterine Bleeding. Danforth's Obstetrics and Gynecology. 9th Edn., Lippincott Williams and Wilkins, ISBN: 0-7817-3730-3, pp: 643-644.
Charles, R., 1999. Dysfunctional Uterine Bleeding. Dewhursts Text Book of Obstetrics and Gynecology for Post Graduates. 6th Edn., John Wiley and Sons, ISBN: 0766526511, pp: 409-411.

Davey, D.A., 1995. Dysfunctional Uterine Bleeding. Dewhursts Text Book of Obstetrics and Gynecology for Post Graduates. 5th Edn., John Wiley and Sons, USA., pp: 590-593.

Farn, R., 2000. Abnormal Uterine Bleeding. Gynecology by 10 Teachers. 17th Edn., Oxford University Press, Oxford, pp: 54-55.

Geeta, N., 2000. Abnormal Uterine Bleeding. Gynecology by 10 Teachers. 17th Edn., Oxford University Press, Oxford, ISBN: 13: 978-0-34081664-2, pp: 48-51.

Hallberg, L., 1995. Normal and Abnormal Menstruation. Dewhursts Text book of Obstetrics and Gynecology for post Graduates. 5th Edn., John Wiley and Sons, ISBN: 085542652, pp: 590-595.

Jacobs, A.J., 1995. Incidence of Missed Organic Disease. Dewhursts Text Book of Obstetrics and Gynecology for Post Graduates. 5th Edn., John Wiley and Sons, ISBN: 085542652, pp: 590-592.

Jane, E., 2000. Abnormal Uterine Bleeding. Gynecology by 10 Teachers. 17th Edn., Oxford University Press, ISBN: 13: 978-0-340-81564-2, pp: 51-54.

James, R., 2003. Abnormal Uterine Bleeding. Danforth's Obstetrics and Gynecology. 9th Edn., Lippincott Williams and Wilkins, ISBN: 0-56433830-2, pp: 649-650.

Matthew, C., 2003. Endocrine Disorders. Berek and Novak's Gynecology. 14th Edn., Lippincott Williams and Wilkins, pp: 1076-1080.

Suther Land, M., 1995. Incidence and Effects of Age and Parity. Dewhursts Text book of Obstetrics and Gynecology for post Graduates. 5th Edn., John Wiley and Sons, ISBN: 085542652, pp: 592-594.

Stuartc, C., 2000. Disorders of the Menstrual Cycle. Gynecology by 10 Teachers. 17th Edn., John Wiley and Sons, ISBN: 0665426511, pp: 48-50.

Steve, N., 2003. Abnormal Uterine Bleeding. Danforth's Obstetrics and Gynecology. 9th Edn., Lippincott Williams and Wilkins, pp: 48-50.

Van, E., 1995a. Blood Loss. Dewhursts Text Book of Obstetrics and Gynecology for post Graduates. 5th Edn., John Wiley and Sons, pp: 591-594.

Van, E., 1995b. Definition and Classification of Dysfunctional Uterine Bleeding: Dewhursts Text Book of Obstetrics and Gynecology for Post Graduates. 5th Edn., John Wiley and Sons, USA., ISBN: 085542652, pp: 590-591.

Whitehead, M.I., 1999. Menopanse. Dewhurst's Text book of Obstetrics and Gynecology for post Graduates. 6th Edn., John Wiley and Sons, USA., ISBN: 0765426511, pp: 453-454. 